\title{
The hydrolysis of 4-amino- and 4-dimethylaminobenzene sulfonyl chlorides: an unprecedented case of independence of reactivity from $\mathrm{pH}$
}

Giorgio Cevasco, ${ }^{\dagger *}$ Anna Piątek, ${ }^{\dagger}$ and Sergio Thea ${ }^{\dagger *}$

$\dagger$ Department of Chemistry and Industrial Chemistry, University of Genova, Via Dodecaneso, 31 16146 Genova, Italy

$\S C N R$ - Institute of Chemical Methodologies, Roma, Italy

sergio.thea@unige.it; giorgio.cevasco@unige.it

General. Starting solvents and reagents were of analytical reagent grade or were redistilled or recrystallized from bench quality materials. Deionized water was distilled from glass and preboiled prior to use to free it from dissolved carbon dioxide. Dioxane (Fluka, UV spectroscopic grade) was dried and freed from peroxides by passage through an activated alumina column, and the absence of peroxides was assessed by the KI test. UV-Vis spectra were recorded with a Kontron Uvikon 941 spectrophotometer.

Materials. Literature methods were followed for the synthesis of 4-aminobenzenesulfonyl chloride 1 and the corresponding hydrochloride $\mathbf{1} \cdot \mathbf{H C l}^{1}, 4$-aminobenzenesulfonyl fluoride $\mathbf{2}^{2}$, and 4dimethylaminobenzenesulfonyl chloride $4^{3}$. Purity was assessed by measuring their m.p.s: $1 \cdot \mathrm{HCl}$ : m.p. 236-238 ${ }^{\circ} \mathrm{C}$ (decomp.), (lit. $238^{\circ} \mathrm{C}$, decomp.) ${ }^{1}$ (compound 1 does not possess a distinct melting point as it undergoes polymerization on heating; its I.R. spectrum, however was identical to that reported by previous authors ${ }^{1}$ ), 2: m.p. $70-71{ }^{\circ} \mathrm{C}$ (lit. 70) ${ }^{2}$, 4: m.p. $109-110^{\circ} \mathrm{C}$ (lit. $109.5-111.0$ ). ${ }^{3}$ All compounds gave ${ }^{1}$ H N.M.R. spectra fully consistent with their structures. As seen in preliminary experiments, chloride $\mathbf{1}$ and its hydrochloride $\mathbf{1} \cdot \mathbf{H C l}$, as expected, at given $\mathrm{pH}$ showed the same reactivity. As $\mathbf{1} \cdot \mathbf{H C l}$ is far more stable than $\mathbf{1}$ (it can be stored unaltered in a dessiccator for long periods of time, and stock dioxane solutions of it are quite stable as well), in most experiments it was employed instead of the free base.

Kinetic measurements. In the case of the least reactive sulfonyl fluoride 2, kinetic measurements were performed with the Kontron Uvikon 941 spectrophotometer; the same machine equipped with a SFA11 Rapid Kinetic accessory (HI-TEC SCIENTIFIC Ltd.) was employed to follow the hydrolyses of the more reactive chlorides $\mathbf{1}$ and $\mathbf{4}$ in 20\% aqueous dioxane; very fast hydrolysis of the chloride $\mathbf{1}$ in water solvent was followed by means of a stopped-flow apparatus equipped with a parallel diode-array detector.

When the standard kinetic tecnique was used (as in the case of chloride 2), the buffered solution (2.5 $\mathrm{mL})$ was equilibrated to the required temperature $\left( \pm 0.1^{\circ} \mathrm{C}\right)$ in a 1-cm path-length quartz cell placed in the thermostated cell holder of the Kontron Uvikon spectrophotometer. The reaction was initiated by adding 25 or $50 \mu \mathrm{L}$ of a stock solution of the substrate ca. $0.01 \mathrm{M}$ in dioxane to the buffer, and automated acquisition of 50-200 data points for each kinetic run was performed. In all cases at least three different buffer concentrations, at constant $\mathrm{pH}$, were employed. The ionic strength was kept at 1.0 $\mathrm{M}$ with $\mathrm{KCl}$. The $\mathrm{pH}$ of the buffered solutions were measured before and after each kinetic run using a Ross combined electrode, calibrated with standard buffers. All $\mathrm{pH}$ values quoted for the dioxane-water solutions are relative values measured directly, no further corrections being applied. The pseudo-firstorder rate constants $\left(\mathrm{k}_{\mathrm{obs}}\right)$ were obtained by NLLSQ fitting of absorbance vs time data, ${ }^{4}$ and the values reported in Table $\mathrm{S} 2$ are the averages of at least duplicate runs.

Reactions were normally followed over about eigth half-lives. The rate constants for the hydrolysis of 2 at $\mathrm{pH}$ values below 12 were obtained by initial rates: they were measured for each run up to ca. $10 \%$ of 
the total reaction and were converted to pseudo-first-order rate constants using infinity values calculated from the known extinction coefficients of the products.

Kinetic experiments for the fast reactions of sulfonyl chlorides $\mathbf{1}$ and $\mathbf{4}$ in $20 \%$ aqueous dioxane solvent were carried out with the Kontron Uvikon instrument equipped with the SFA-11 Rapid Kinetic accessory. Temperature control was ensured by injecting the pre-thermostatted solutions into the flow cell inserted into the thermostatted cell holder of the spectrophotometer. Rate constants were assessed by following the absorbance decrease at $309 \mathrm{~nm}$, due to the disappearance of the substrates. ${ }^{4}$

Kinetic experiments for the very fast reactions of chloride $\mathbf{1}$ in water solvent were run on the diodearray stopped-flow apparatus. Reaction course was followed monitoring the disappearance of the substrate absorption band in the 270 - $340 \mathrm{~nm}$ interval, whilst rate constants were obtained from the decrease of absorbance at a single, selected wavelength in the range $295.5-320.5 \mathrm{~nm}$. Temperature control was assured by the mixing device of the apparatus, consisting of a SF-3L Hi-Tech support unit. Calculations were carried out by means of the SPECFIT program.

\section{References}

(1) Contreras, J.; Jones, J.I. Brit. Polym. J. 1980, 192 - 198.

(2) De Cat A.; Van Poucke R. J. Org. Chem. 1963, 28, 3426.

(3) Sukenik, C.N.; Bonapace, J.A.P.; Mandel, N.S.; Pui-Yan Lau, Wood, G.; Bergman, R. G. J. Am. Chem. Soc. 1977, 99, $851-858$.

(4) The values of the kinetic parameters, as well as the $\mathrm{p} K_{\mathrm{a}}$ values, were obtained from primary kinetic data by iterative nonlinear curve-fitting performed with the Fig.P program (Biosoft, Cambridge, UK).

(5) Harned, S.; Fallon, L. D. J. Am. Chem. Soc. 1939, 61, 2374-2377. 
Table S1. Hydrolysis of 4-aminobenzenesulfonyl chloride. Solvent water, ionic st rength $=1 \mathrm{~N}$ (kept constant with $\mathrm{KCl}) ; \mathrm{t}=(25.0 \pm 0.1)^{\circ} \mathrm{C}$; stopped flow kinetics;analytical wavelength 308.5 nm.

\begin{tabular}{llll}
\hline Buffer & $\mathrm{pH}$ & $\mathrm{k}_{\mathrm{obs}}\left(\mathrm{s}^{-1}\right)$ & $\log \mathrm{k}_{\mathrm{obs}}$ \\
\hline KOH 0.1 N & 13.00 & $81.5 \pm 6.0$ & 1.91 \\
$\mathrm{KOH} 0.05 \mathrm{~N}$ & 12.70 & $52.1 \pm 4.0$ & 1.72 \\
$\mathrm{KOH} \mathrm{0.01} \mathrm{N}$ & 12.00 & $9.65 \pm 0.50$ & 0.98 \\
KOH 0.005 N & 11.70 & $7.80 \pm 0.20$ & 0.89 \\
Carbonate & 9.75 & $5.20 \pm 0.20$ & 0.72 \\
Citrate & 6.00 & $6.60 \pm 0.20$ & 0.82 \\
HCl 0.001 N & 3.00 & $4.50 \pm 0.10$ & 0.65 \\
HCl 0.01 N & 2.00 & $4.50 \pm 0.20$ & 0.65 \\
HCl 0.05 N & 1.30 & $4.55 \pm 0.20$ & 0.66 \\
HCl 0.1 N & 1.00 & $4.10 \pm 0.20$ & 0.61 \\
HCl 1 N & 0.00 & $0.90 \pm 0.10$ & -0.05 \\
\hline
\end{tabular}

Derived parameters (FigP) are: $k_{\mathrm{a}}=(5.17 \pm 0.40) \mathrm{s}^{-1} ; k_{\mathrm{b}}=(740 \pm 90) \mathrm{M}^{-1} \mathrm{~s}^{-1} ; \mathrm{p} K_{\mathrm{a}}=0.64 \pm 0.10$ 
Table S2. Hydrolysis of 4-aminobenzenesulfonyl fluoride. Solvent water, ionic strength $=1 \mathrm{~N}$ (kept constant with $\mathrm{KCl}) ; \mathrm{t}=(25.0 \pm 0.1){ }^{\circ} \mathrm{C}$; analytical wavelength: $275 \mathrm{~nm}$.

\begin{tabular}{llll}
\hline Buffer & $\mathrm{pH}$ & $\mathrm{k}_{\text {obs }}\left(\mathrm{s}^{-1}\right)$ & $-\log \mathrm{k}_{\mathrm{obs}}$ \\
\hline KOH 0.0984 N & 12.99 & $3.56 \cdot 10^{-2}$ & 1.449 \\
$\mathrm{KOH} \mathrm{0.0738} \mathrm{N}$ & 12.87 & $2.84 \cdot 10^{-2}$ & 1.547 \\
$\mathrm{KOH} \mathrm{0.0492} \mathrm{N}$ & 12.69 & $1.93 \cdot 10^{-2}$ & 1.714 \\
KOH 0.0246 N & 12.39 & $1.04 \cdot 10^{-2}$ & 1.983 \\
KOH 0.01 N & 12.00 & $3.47 \cdot 10^{-3}$ & 2.460 \\
carbonate & 10.06 & $4.42 \cdot 10^{-5}$ & 4.355 \\
phosphate & 8.02 & $5.30 \cdot 10^{-7}$ & 6.278 \\
\hline
\end{tabular}

Derived parameters $(\mathrm{FigP})$ are: $k_{\mathrm{OH}}=(3.67 \pm 0.11) \cdot 10^{-1} \mathrm{M}^{-1} \mathrm{~s}^{-1}$ 
Table S3. Hydrolysis of 4-aminobenzenesulfonyl chloride. Solvent $20 \%$ aqueous dioxane (v/v), ionic strength $=1 \mathrm{~N}$ (kept constant with $\mathrm{KCl}) ; \mathrm{t}=(25.0 \pm 0.1){ }^{\circ} \mathrm{C}$; stopped flow kinetics; analytical wavelength range $250-330 \mathrm{~nm} ; \mathrm{p} K_{\mathrm{w}}=14.51^{\mathrm{a}}$

\begin{tabular}{llll}
\hline Buffer & $\mathrm{pH}$ & $\mathrm{k}_{\text {obs }}\left(\mathrm{s}^{-1}\right)$ & $\log \mathrm{k}_{\mathrm{obs}}$ \\
\hline KOH 0.01 N & 12.51 & $2.73 \pm 0.10$ & -0.436 \\
KOH 0.005 N & 12.21 & $1.73 \pm 0.03$ & -0.238 \\
phosphate & 11.64 & $1.15 \pm 0.14$ & -0.061 \\
carbonate & 10.38 & $0.65 \pm 0.03$ & 0.194 \\
ammonia & 9.38 & $0.57 \pm 0.10$ & 0.244 \\
phosphate & 6.96 & $0.64 \pm 0.03$ & 0.194 \\
citrate & 5.00 & $0.67 \pm 0.02$ & 0.171 \\
citrate & 4.55 & $0.57 \pm 0.05$ & 0.244 \\
HCl $0.01 \mathrm{~N}$ & 2.10 & $0.61 \pm 0.01$ & 0.218 \\
$\mathrm{HCl} 0.05 \mathrm{~N}$ & 1.40 & $0.58 \pm 0.01$ & 0.237 \\
$\mathrm{HCl} 0.1 \mathrm{~N}$ & 1.10 & $0.57 \pm 0.01$ & 0.243 \\
$\mathrm{HCl} 0.5 \mathrm{~N}$ & 0.40 & $0.47 \pm 0.01$ & 0.327 \\
$\mathrm{HCl} 1 \mathrm{~N}$ & 0.10 & $0.40 \pm 0.01$ & 0.402 \\
\hline
\end{tabular}

(a) Value is from Ref. 5.

Derived parameters (FigP) are: $k_{\mathrm{a}}=(0.62 \pm 0.02) \mathrm{s}^{-1} ; k_{\mathrm{b}}=(318 \pm 27) \mathrm{M}^{-1} \mathrm{~s}^{-1}$ 
Table S4. Hydrolysis of 4-dimethyaminobenzenesulfonyl chloride. Solvent $20 \%$ aqueous dioxane $(\mathrm{v} / \mathrm{v})$, ionic strength $=1 \mathrm{~N}$ (kept constant with $\mathrm{KCl}) ; \mathrm{t}=(25.0 \pm 0.1){ }^{\circ} \mathrm{C}$; stopped flow kinetics; analytical wavelength range $250-330 \mathrm{~nm} ; \mathrm{p} K_{\mathrm{w}}=14.51^{\mathrm{a}}$

\begin{tabular}{llll}
\hline Buffer & $\mathrm{pH}$ & $k_{\mathrm{obs}}\left(\mathrm{s}^{-1}\right)$ & $\log k_{\mathrm{obs}}$ \\
\hline KOH 1 N & 14.51 & $2.78 \pm 0.09$ & 0.444 \\
KOH 0.1 N & 13.51 & $2.52 \pm 0.23$ & 0.401 \\
KOH 0.01 N & 12.51 & $2.47 \pm 0.08$ & 0.393 \\
carbonate & 10.31 & $2.39 \pm 0.15$ & 0.397 \\
ammonia & 9.38 & $2.37 \pm 0.16$ & 0.375 \\
phosphate & 6.93 & $2.36 \pm 0.13$ & 0.373 \\
citrate & 5.17 & $2.54 \pm 0.09$ & 0.405 \\
HCl 0.001 N & 3.10 & $2.27 \pm 0.07$ & 0.356 \\
HCl 0.01 N & 2.10 & $2.36 \pm 0.16$ & 0.373 \\
HCl 0.1 N & 1.10 & $2.46 \pm 0.26$ & 0.391 \\
HCl 1 N & 0.10 & $2.52 \pm 0.16$ & 0.401 \\
HCl 2.4 N & -0.278 & $1.11 \pm 0.02$ & 0.041 \\
\hline
\end{tabular}

(a) Value is from Ref. 5

(b) This value is not shown in Figure 1 because ionic strength was $\neq 1 \mathrm{M}$ (see text)

Derived parameters (FigP) are: $\mathrm{k}_{\mathrm{a}}=(2.46 \pm 0.04) \mathrm{s}^{-1}$ 


\section{Supporting Information page 7/19}

Table S5. Rate data for calculation of activation parameters ${ }^{a}$ for the hydrolysis of 4aminobenzenesulfonyl chloride in $20 \%$ aqueous dioxane $(\mathrm{v} / \mathrm{v})$; ionic strength $=1 \mathrm{M}(\mathrm{KCl})$; stopped flow kinetics; analytical wavelength range $250-330 \mathrm{~nm}$.

\begin{tabular}{llll}
\hline $\mathbf{p H}$ & $\mathbf{t}\left({ }^{\circ} \mathrm{C}\right)$ & $\boldsymbol{k}_{\text {obs }}\left(\mathrm{s}^{-1}\right)$ & \\
\hline & 17.1 & $0.38 \pm 0.03$ & \\
& 25.0 & $0.64 \pm 0.03$ & $\Delta \mathrm{H}^{\neq}=(44.5 \pm 0.2) \mathrm{kJ} \mathrm{mol}^{-1} ;$ \\
& 34.9 & $1.17 \pm 0.10$ & $\Delta \mathrm{S}^{\neq}=(-99.6 \pm 0.6) \mathrm{J} \mathrm{K}^{-1} \mathrm{~mol}^{-1}$ \\
& 16.9 & $0.94 \pm 0.10$ & \\
& 25.0 & $1.73 \pm 0.03$ & $\Delta \mathrm{H}^{\neq}=(46.00 \pm 2.22) \mathrm{kJ} \mathrm{mol}^{-1}$ \\
$12.21^{\mathrm{c}}$ & 34.0 & $2.88 \pm 0.30$ & $\Delta \mathrm{S}^{\neq}=\left(-86.6 \pm 7.5 \mathrm{~J} \mathrm{~K}^{-1} \mathrm{~mol}^{-1}\right)$ \\
\hline
\end{tabular}

(a) $\Delta \mathrm{S}^{\not \neq}$ values were calculated at $25^{\circ} \mathrm{C}$

(b) phosphate buffer

(c) $0.005 \mathrm{M} \mathrm{KOH}$ 


\section{Supporting Information page 8/19}

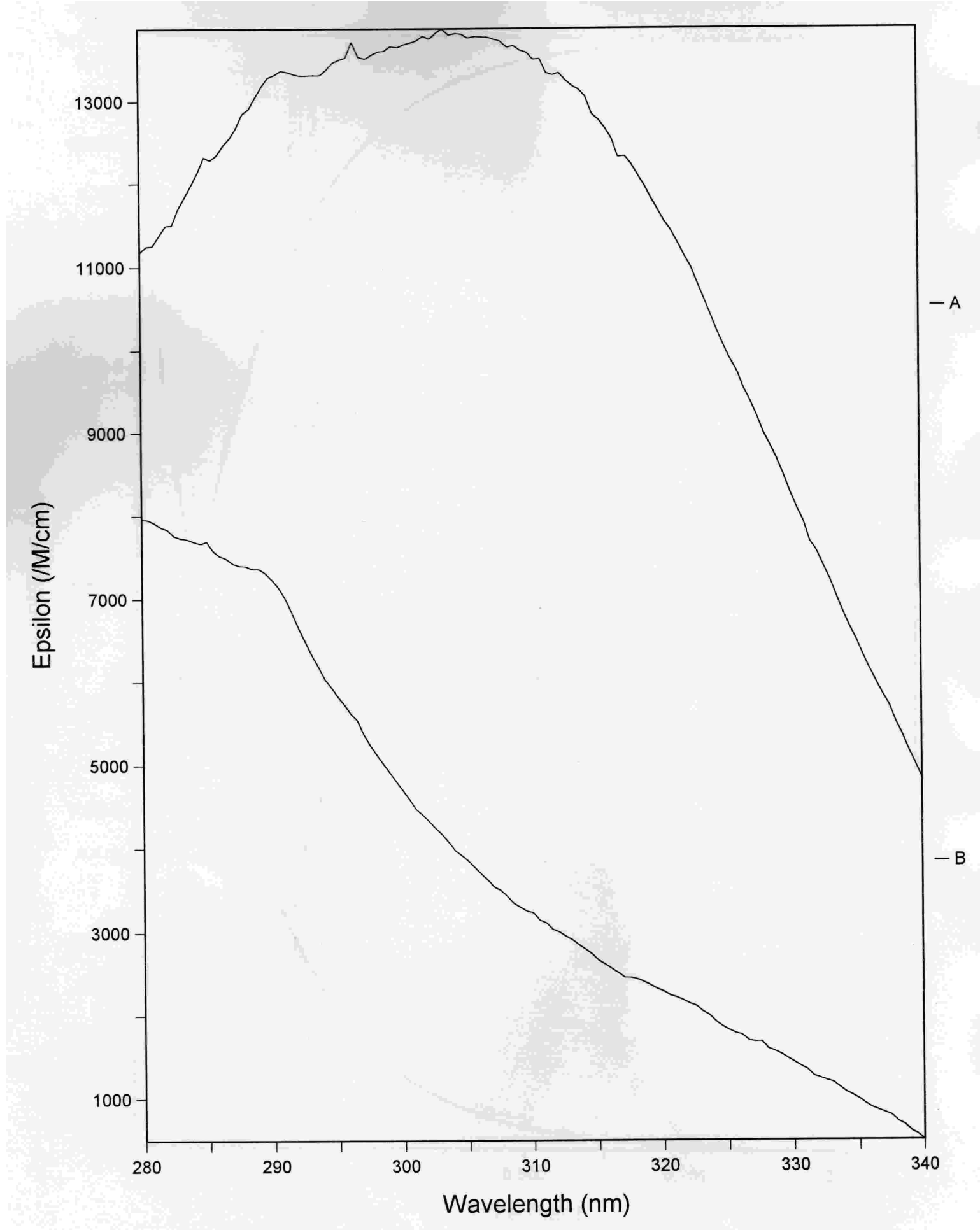

Figure S1. Hydrolysis of 4-aminobenzenesulfonyl chloride 1, initial concentration $2.5 \times 10^{-5} \mathrm{M}$. Solvent water, $\mathrm{pH} 11.70(\mathrm{KOH})$, ionic strength $1 \mathrm{M}(\mathrm{KCl})$, temperature $(25 \pm 0.1)^{\circ} \mathrm{C}$. "Zero time" (upper curve) and "infinity" (lower curve) spectra are shown. 


\section{Supporting Information page 9/19}
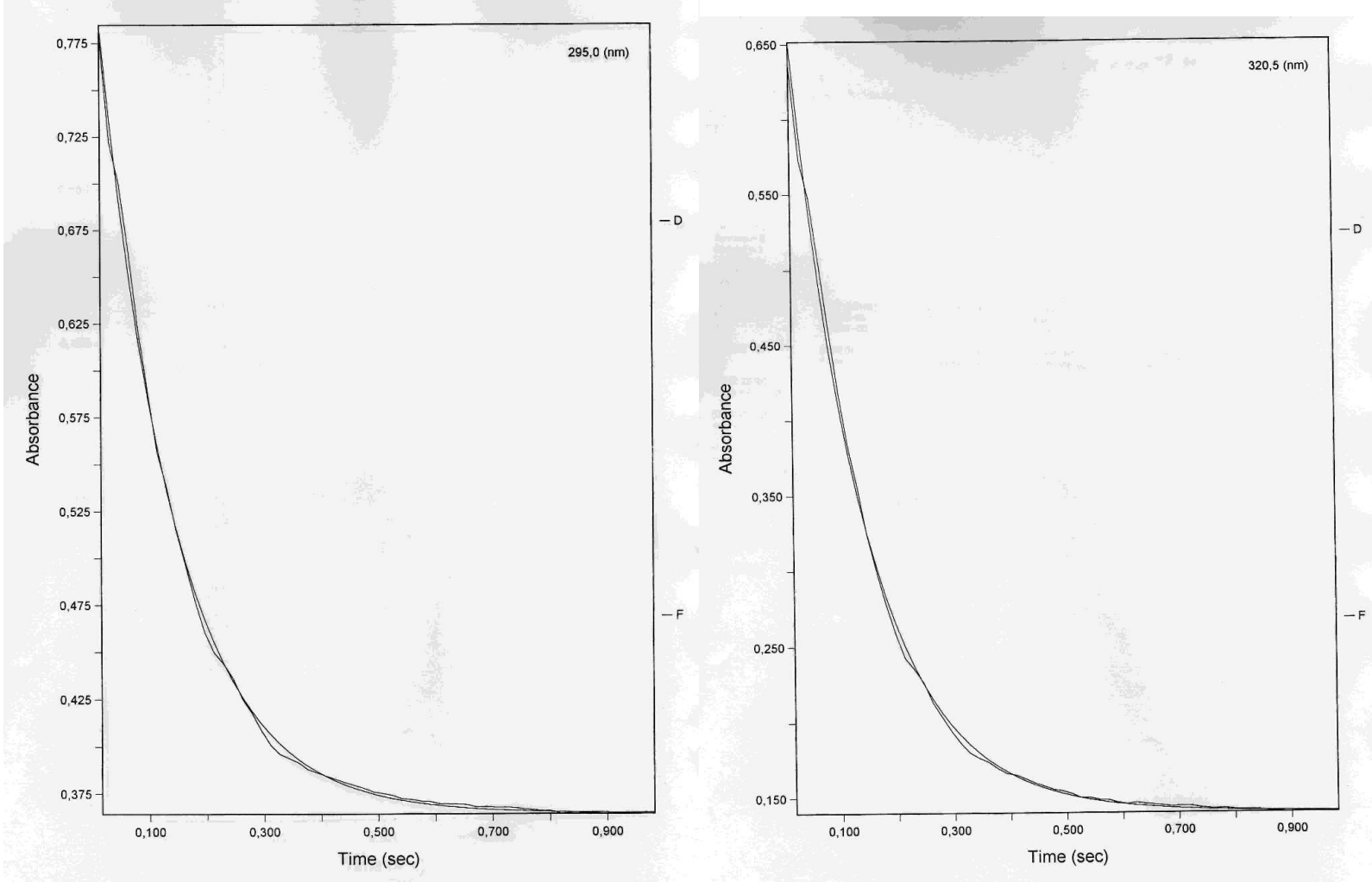

Figure S2. Absorbance vs. time plots (experimental and theoretical) for the hydrolysis of 4-aminobenzenesulfonate 1.Analytical wavelengths 295.0 and $320.5 \mathrm{~nm}$. Other conditions are the same as those given in Figure S1. 


\section{Supporting Information page 10/19}

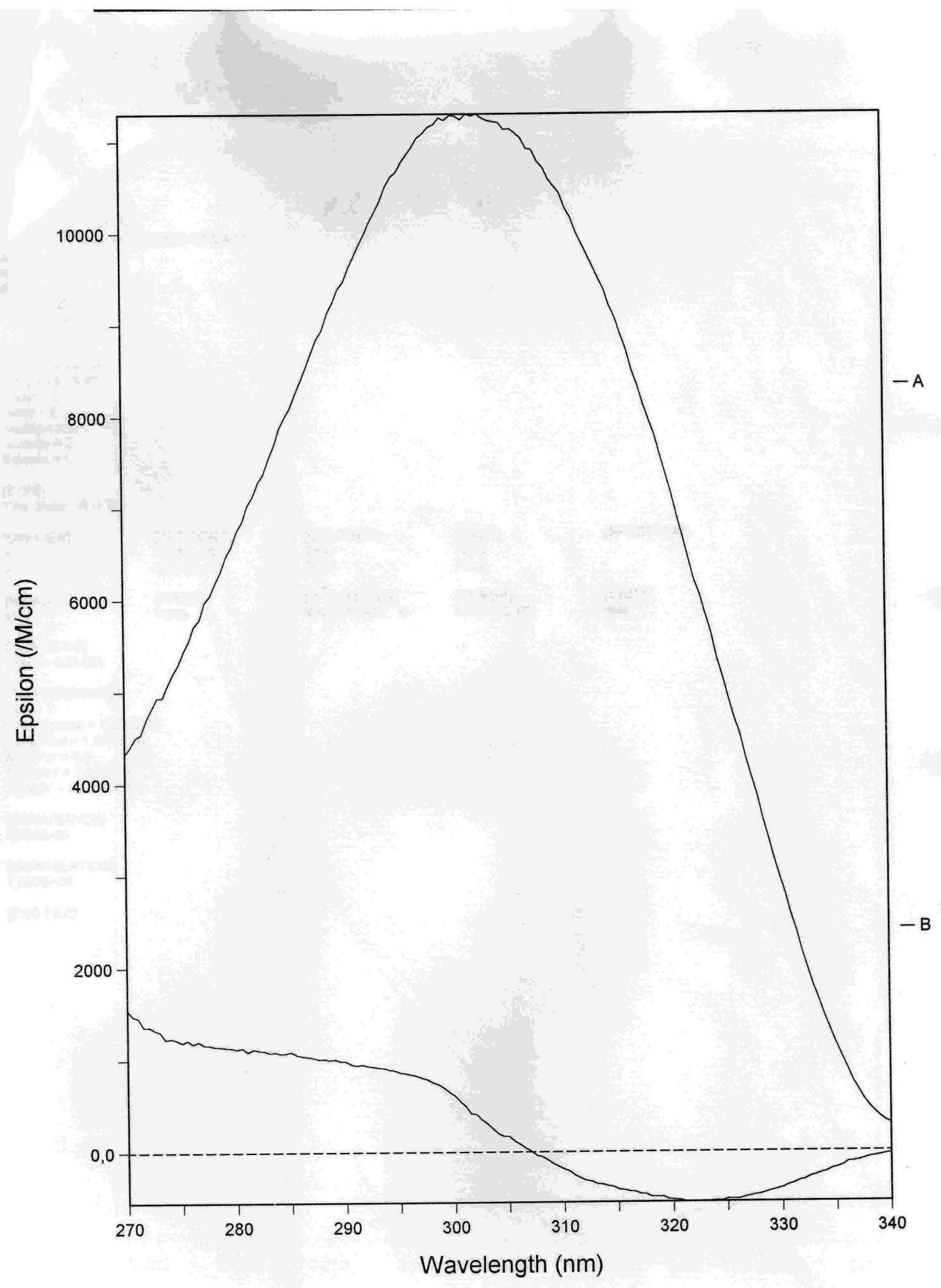

Figure S3. Hydrolysis of 4-aminobenzenesulfonyl chloride 1, initial concentration $2.5 \times 10^{-5} \mathrm{M}$. Solvent water, $\mathrm{pH} 1.30(\mathrm{HCl})$, ionic strength $1 \mathrm{M}(\mathrm{KCl})$, temperature $(25 \pm 0.1){ }^{\circ} \mathrm{C}$. "Zero time" (upper curve) and "infinity" (lower curve) spectra are shown. 
Supporting Information page 11/19

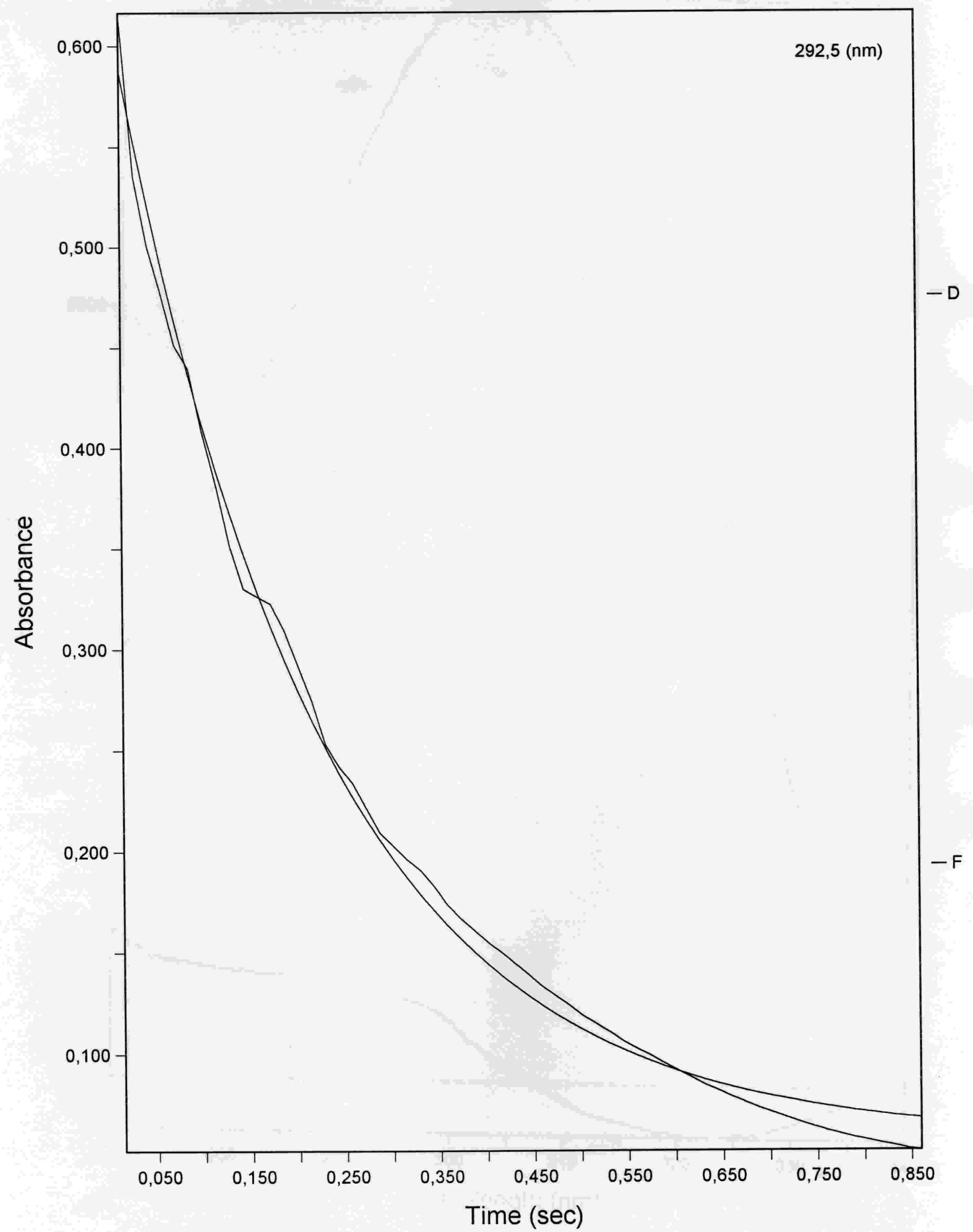

Figure S4. Absorbance vs. time plots (experimental and theoretical)for the hydrolysis of 4-aminobenzenesulfonate 1.Analytical wavelength $292.5 \mathrm{~nm}$. Other conditions are the same as those given in Figure S3. 
Supporting Information page 12/19

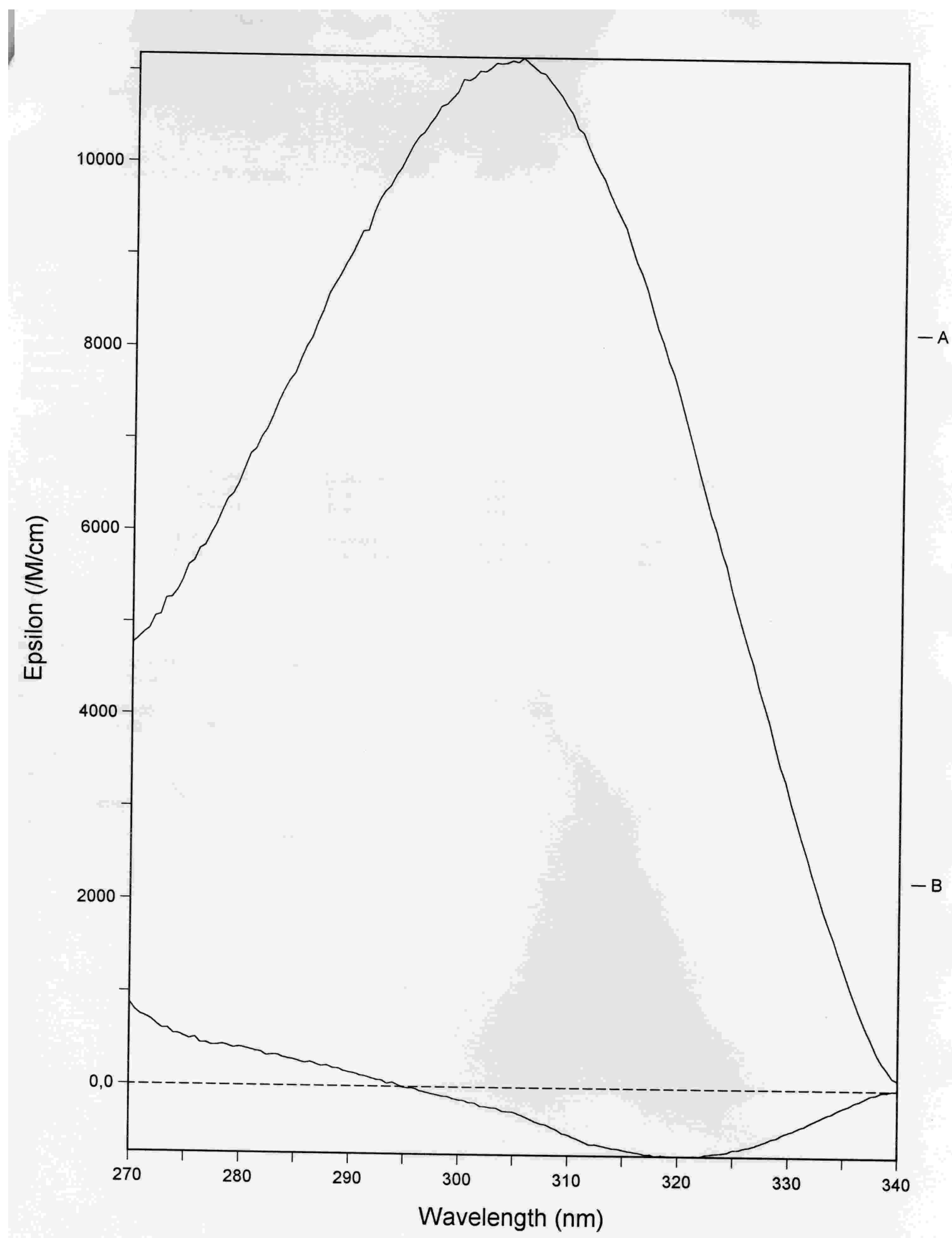

Figure S5. Hydrolysis of 4-aminobenzenesulfonyl chloride 1, initial concentration $2.5 \times 10^{-5} \mathrm{M}$. Solvent water, $\mathrm{pH} 3.00(\mathrm{HCl})$, ionic strength $1 \mathrm{M}(\mathrm{KCl})$, temperature $(25 \pm 0.1){ }^{\circ} \mathrm{C}$. "Zero time" (upper curve) and "infinity" (lower curve) spectra are shown. 
Supporting Information page 13/19
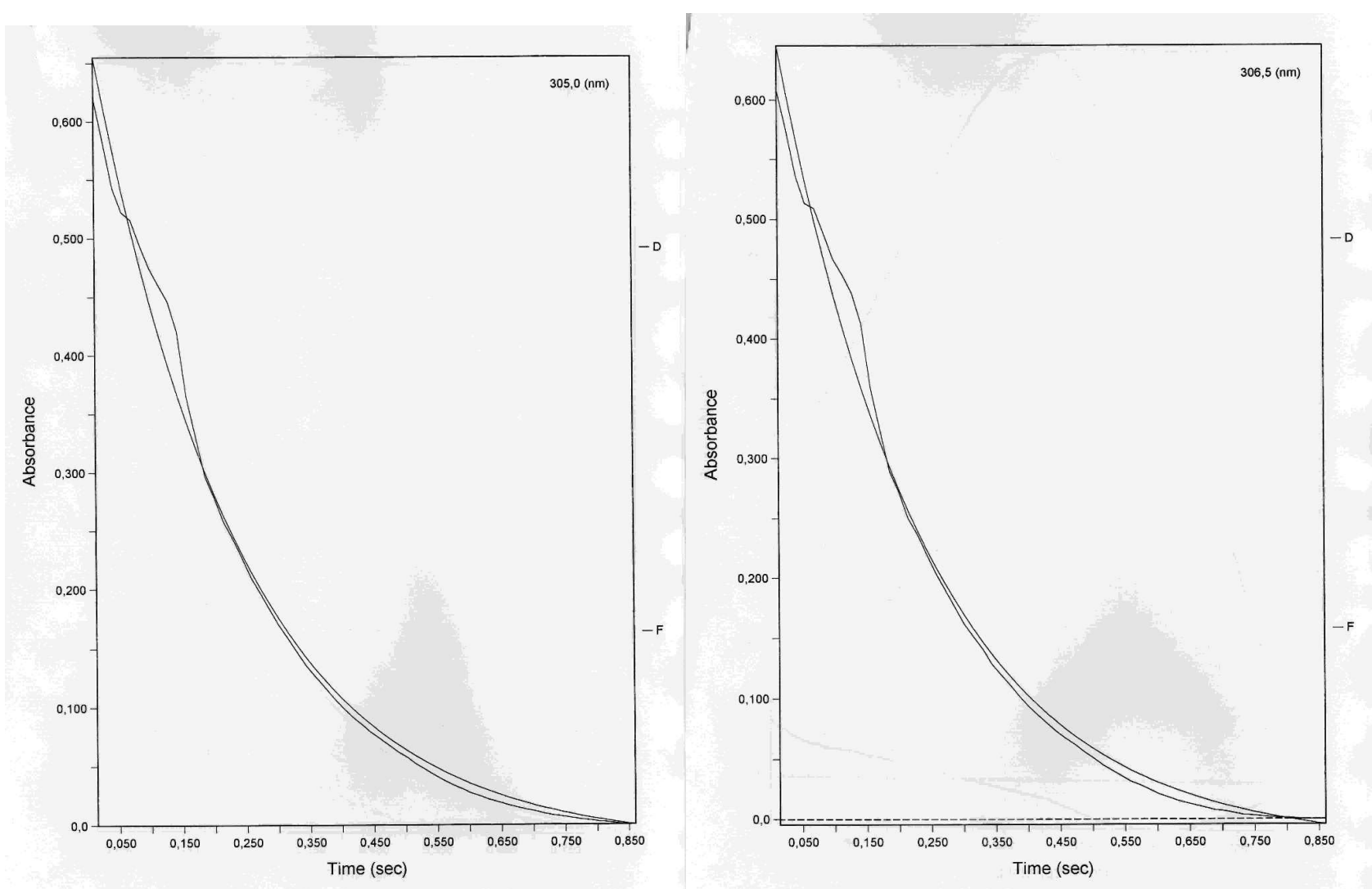

Figure S6. Absorbance $v s$. time plots (experimental and theoretical) for the hydrolysis of 4-aminobenzenesulfonyl chloride 1. Analytical wavelengths 305.0 and $306.5 \mathrm{~nm}$. Other conditions are the same as those given in Figure S5. 
Supporting Information page 14/19

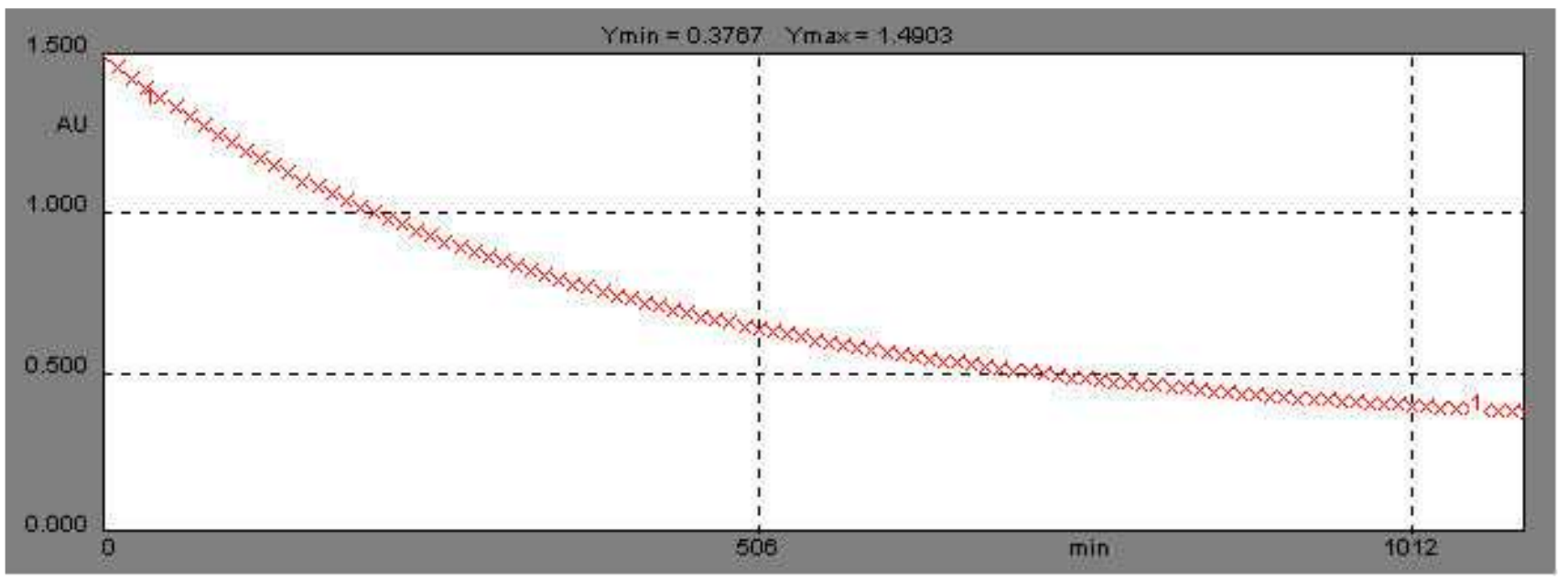

Figure S7. Absorbance vs. time plot for the hydrolysis of 4-aminobenzenesulfonyl fluoride 2. Solvent water, $\mathrm{pH}=10.06$ (carbonate buffer), ionic strength $1.0 \mathrm{M}(\mathrm{KCl})$, temperature $(25 \pm 0.1){ }^{\circ} \mathrm{C}$, analytical wavelength $275 \mathrm{~nm}$. 
Supporting Information page 15/19

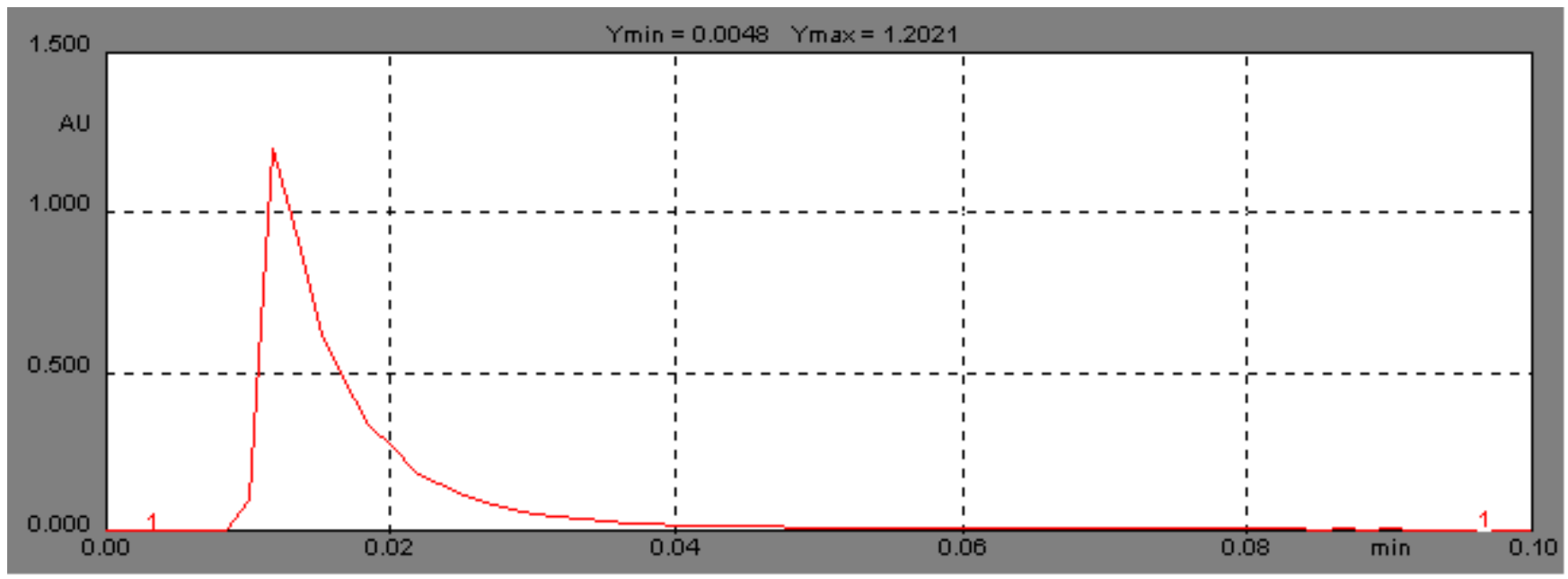

Figure S8. Absorbance vs. time plot for the hydrolysis of 4-aminobenzenesulfonyl chloride 1 . Solvent $20 \%$ aqueous dioxane, $\mathrm{pH}=12.51$ $(\mathrm{KOH})$, ionic strength $1.0 \mathrm{M}(\mathrm{KCl}), \mathrm{p} K_{\mathrm{w}}=14.51$, temperature $(25 \pm 0.1){ }^{\circ} \mathrm{C}$, analytical wavelength $309 \mathrm{~nm}$. 


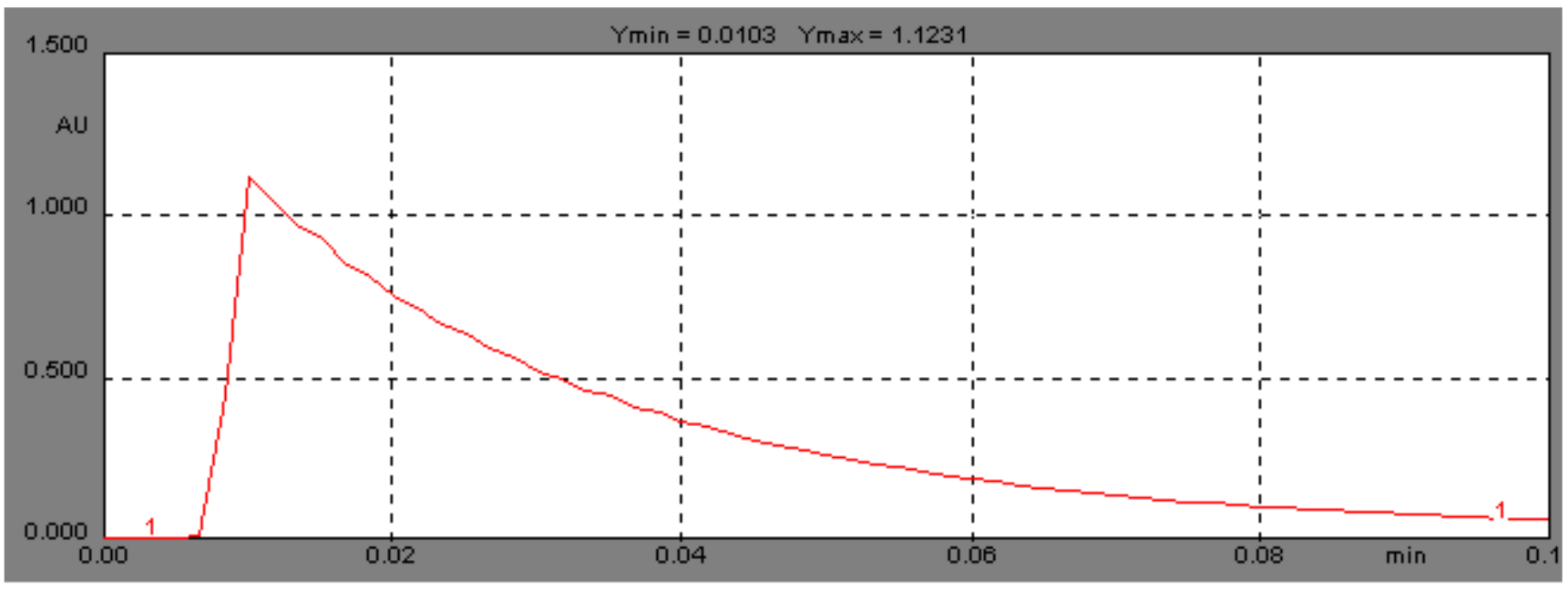

Figure S9. Absorbance vs. time plot for the hydrolysis of 4-aminobenzenesulfonyl chloride $\mathbf{1}$. Solvent $20 \%$ aqueous dioxane, $\mathrm{pH}=6.96$ (phosphate buffer), ionic strength $1.0 \mathrm{M}(\mathrm{KCl}), \mathrm{p} K_{\mathrm{w}}=14.51$, temperature $(25 \pm 0.1){ }^{\circ} \mathrm{C}$, analytical wavelength $309 \mathrm{~nm}$. 
Supporting Information page 17/19

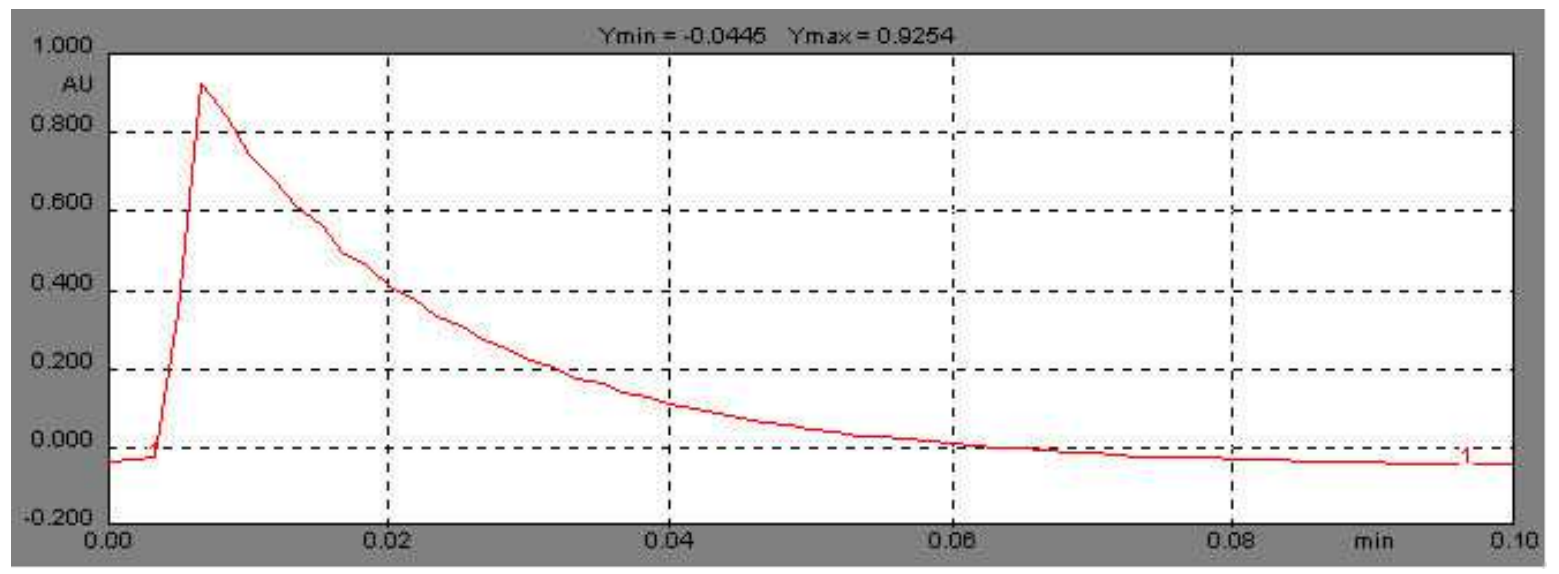

Figure S10. Absorbance vs. time plot for the hydrolysis of 4-aminobenzenesulfonyl chloride 1. Solvent $20 \%$ aqueous dioxane, $\mathrm{pH}=11.64$ (phosphate buffer), ionic strength $1.0 \mathrm{M}(\mathrm{KCl}), \mathrm{p} K_{\mathrm{w}}=14.51$, temperature $(25 \pm 0.1){ }^{\circ} \mathrm{C}$, analytical wavelength $309 \mathrm{~nm}$. 
Supporting Information page 18/19

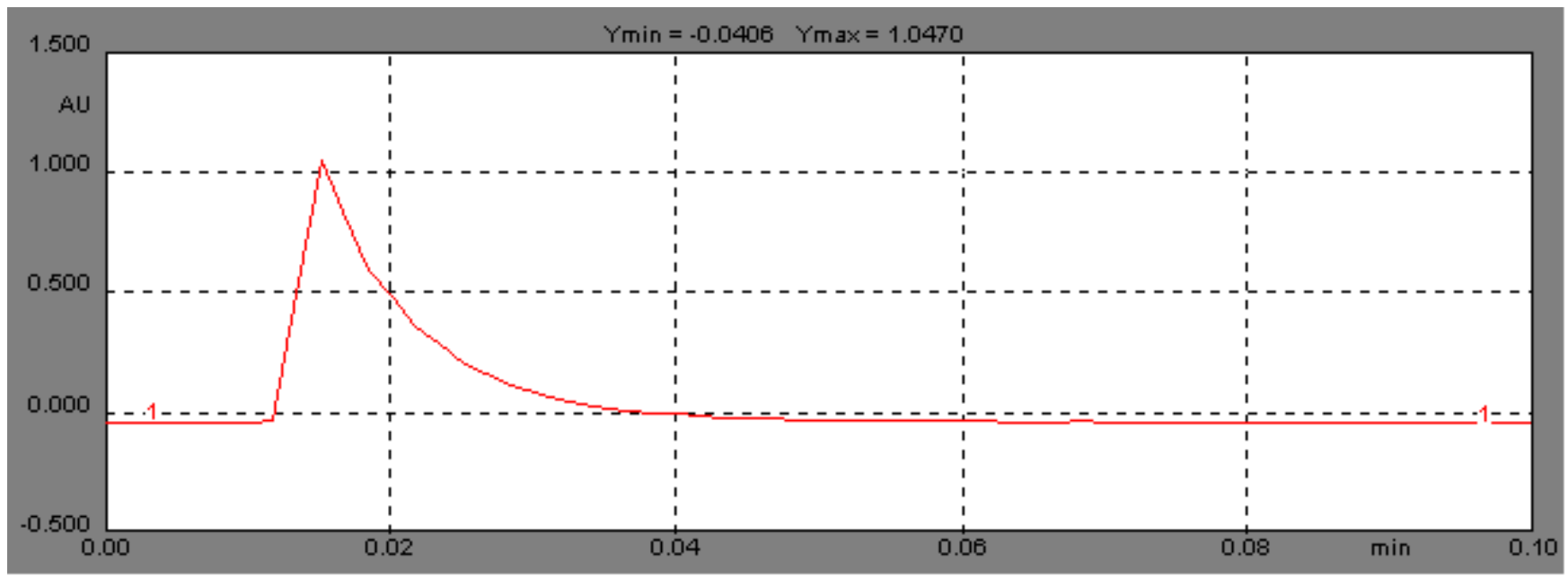

Figure S11. Absorbance vs. time plot for the hydrolysis of 4-dimethylaminobenzenesulfonyl chloride 4. Solvent $20 \%$ aqueous dioxane, $\mathrm{pH}=13.51(\mathrm{KOH})$, ionic strength $1.0 \mathrm{M}(\mathrm{KCl}), \mathrm{p} K_{\mathrm{w}}=14.51$, temperature $(25 \pm 0.1){ }^{\circ} \mathrm{C}$, analytical wavelength $309 \mathrm{~nm}$. 


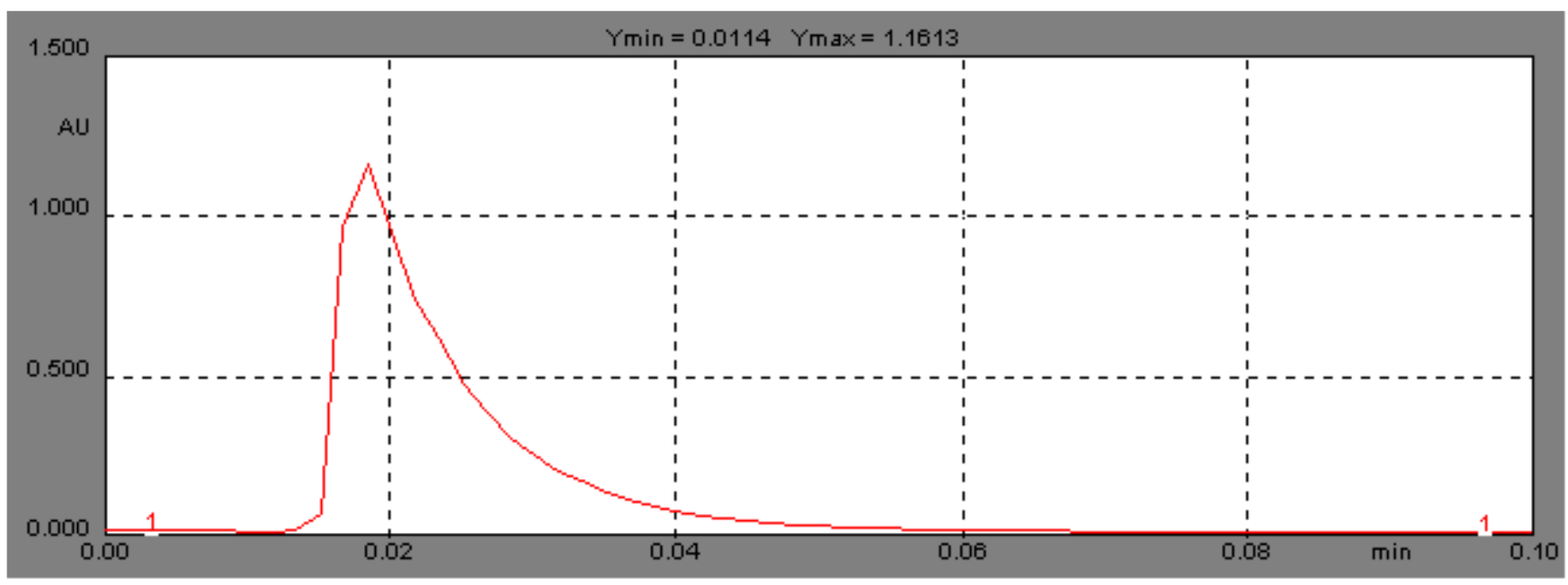

Figure S12. Absorbance vs. time plot for the hydrolysis of 4-dimethylaminobenzenesulfonyl chloride 4. Solvent $20 \%$ aqueous dioxane, $\mathrm{pH}=6.93$ (phosphate buffer), ionic strength $1.0 \mathrm{M}(\mathrm{KCl}), \mathrm{p} K_{\mathrm{w}}=14.51$, temperature $(25 \pm 0.1){ }^{\circ} \mathrm{C}$, analytical wavelength $309 \mathrm{~nm}$. 